\title{
Innovación Tecno-Educativa “Google”. Plataformas Digitales, Datos y Formación Docente
}

\section{“Google” Techno-Educational Innovation. Digital Platforms, Data and Teacher Training}

\author{
Geo Saura ${ }^{1, *}$, Enrique-Javier Díez-Gutiérrez ${ }^{2}$, Pablo Rivera-Vargas ${ }^{3,4}$ \\ ${ }^{1}$ Universidad de Granada, España \\ ${ }^{2}$ Universidad de León, España \\ ${ }^{3}$ Universidad de Barcelona, España \\ ${ }^{4}$ Universidad Andrés Bello, Chile
}

\section{DESCRIPTORES:}

Innovación educativa

Tecnología educativa

Plataformas digitales

Datos

Formación de docentes

\section{RESUMEN:}

Las plataformas digitales están generando los avances más significativos en la innovación educativa global. La pandemia mundial de Covid-19 está acelerando la expansión de las plataformas digitales en educación. Google es la corporación tecnológica que, a través de Google for Education, está liderando la innovación educativa mediante sus plataformas digitales. A partir de este contexto, el objetivo del presente artículo es analizar la innovación en las prácticas educativas y en la formación del profesorado, a través de las plataformas digitales de Google. Desde un enfoque cualitativo basado en la implementación de una etnografía digital y una tecnografía, se han analizado en profundidad ocho sitios web de Google que tienen relación con la formación docente y los datos. Los resultados del estudio muestran que, a través de la API de Classroom, se están generando (1) nuevos procesos de control tecno-educativo, (2) nuevas dinámicas extractivas de los datos y (3) nuevas relaciones rentistas con la industria tecnológica. Junto a ello, se observa que la formación docente para la innovación tecno-educativa en Google se basa en un desarrollo profesional piramidal mediante certificaciones docentes, y en "comunidades educativas Google” que se auto-forman y expanden las herramientas y las lógicas de mercado de la corporación tecnológica.

\section{KEYWORDS:}

Educational innovations

Educational technology

Digital platforms

Data

Teacher training

\section{ABSTRACT:}

Digital platforms are generating the most significant advances in global educational innovation. The global pandemic of Covid-19 is accelerating the expansion of digital platforms in education. Google is the technology corporation that, through Google for Education, is leading educational innovation through its digital platforms. In this context, the objective of this article is to analyze innovation in educational practices and teacher training through Google's digital platforms. From a qualitative approach based on the implementation of a digital ethnography and a technography, eight Google websites related to teacher training and data have been analyzed in depth. The results of the study show that, through the Classroom API (1) new techno-educational control processes are being generated, (2) new extractive dynamics of data and (3) new relationships are forming with the technology industry. Alongside this, it is observed that teacher training for techno-educational innovation in Google is based on pyramidal professional development, through teaching certifications, and on "Google educational communities" that are self-formed and expand the tools and market logics of this technology corporation.

CÓMO CITAR:

Saura, G., Díez-Gutiérrez, E. J. y Rivera-Vargas, P. (2021). Innovación tecno-educativa "google”. Plataformas digitales, datos y formación docente. REICE. Revista Iberoamericana sobre Calidad, Eficacia y Cambio en Educación, 19(4), 111-124. https://doi.org/10.15366/reice2021.19.4.007

*Contacto: geosaura@ugr.es

ISSN: $1696-4713$

revistas.uam.es/reice
Recibido:

27 de marzo 2021

1a Evaluación: 11 de mayo 2021

2a Evaluación: 19 de junio 202

Aceptado: 14 de julio 2021 


\section{Introducción}

Las plataformas digitales en educación están generando los avances más significativos en la innovación educativa global (Decuypere et al., 2021; Perrotta et al., 2021). Su irrupción en educación es una parte fundamental de cambios sociales más amplios de la "plataformización" de la sociedad (Van Dijck et al., 2019). A su vez, son un elemento central de lo que Srnicek (2016) denomina como "capitalismo de plataformas".

Debido a la pandemia global Covid-19, que de forma acelerada y disruptiva sigue obligando a adoptar formatos de enseñanza no presencial o semi-presencial en la mayoría de los sistemas educativos de todo el mundo, la expansión de las plataformas digitales sigue disparándose, impulsando el relato de la "innovación digital educativa”. Esta expansión no la lideran los gobiernos, los organismos internacionales o los actores políticos públicos, sino las grandes corporaciones tecnológicas (BigTech), que siguen generando nuevos procesos de "gobernanza digital" (Williamson y Hogan, 2021) y nuevos avances de los "imaginarios socio-técnicos” (Jasanoff y Kim, 2009) de la Industria Educativa Tecnológica (Industria EdTech) de Silicon Valley (Means, 2018).

Los defensores de la privatización educativa ven la pandemia global como la gran oportunidad para que nuevos "socios tecnológicos" lideren la innovación digital de los sistemas educativos a través de relaciones de mercado (Tooley et al., 2020). Otros estudios demuestran que esta expansión de la "innovación educativa” a través de plataformas digitales está generando nuevos procesos de gobernanza global, que hacen que los sistemas educativos sean cada vez más privatizados, más dependientes de las BigTech y, por lo tanto, menos democráticos (Williamson y Hogan, 2020).

Dentro de la industria educativa BigTech, son las GAFAM ${ }^{1}$ quienes dominan las plataformas digitales más significativas de la innovación educativa. Algunas voces denuncian que las GAFAM se introducen en los sistemas educativos a través de discursos solucionistas, revestidos de innovación educativa (Morozov, 2015). Junto a las GAFAM, los "unicornios" de la industria de tecnología educativa (EdTech Unicorns)2, aquellas corporaciones que están valorados en los mercados internacionales con más de mil millones de dólares son también grandes beneficiarios de esta megaindustria (HolonIQ, 2021).

A través de ellas pretenden convertirse en una forma casi omnipresente de intermediarios e infraestructuras (Komljenovic, 2021) que recopilan y procesan los datos, el "oro" del siglo XXI (Couldry y Mejias, 2019). Su influencia se da en todos los procesos educativos, desde la infancia hasta la universidad, incluyendo la formación continua y permanente. Estas plataformas digitales en educación están generando el nuevo "rentismo de los datos" que se basa en "la búsqueda de estrategias de innovación diseñadas para capturar o extraer valor a través de la propiedad y el control de los datos como un activo" (Birch et al., 2020 , p. 470). Estas relaciones rentistas de la innovación global son claves en la "extracción” y "convergencia de capital” (Sadowski, 2019) del actual “capitalismo de vigilancia” (Zuboff, 2019).

De toda la industria EdTech, Google es la corporación que está liderando la "innovación educativa global" a través de sus plataformas digitales educativas y mediante las relaciones rentistas que genera con los EdTech Unicorns (Ideland, 2021; Komljenovic, 2021) ${ }^{3}$. No obstante, hasta el momento, hay escasas investigaciones educativas que analicen su papel en educación. Y menos aún, que analicen los nuevos significados que estas plataformas digitales le están ofreciendo a la noción de innovación educativa.

Google for Education es el paquete de servicios que Google -la filial subsidiaria más importante de la multinacional Alphabet- ofrece a la educación a través de varios productos que tienen distintos dominios específicos, finalidades y proveedores de software y hardware. El paquete de software y hardware dedicados a la educación está dentro de lo que se denomina “Google Workspace for Education”, que fue

1 El acrónimo GAFAM (Google, Apple, Facebook, Amazon y Microsoft) hace referencia a las cinco corporaciones tecnológicas occidentales más importantes que están invirtiendo más capital a través de la 'innovación digital' en educación.

2 Hay 20 EdTech “Unicorns” en todo el mundo que han recaudado en la última década más de 14 billones de dólares y están valoradas en 64 billones de dólares.

3 Estas plataformas de Google las utilizan más de 170 millones de usuarios: https://blog.google/outreach-initiatives/ education/google-workspace-for-education/ 
presentado el 17 de febrero de 2021, y que sustituye a lo que desde el año 2006 se había venido designando como "Google G-Suite for Education".

Workspace for Education es un conjunto de herramientas y plataformas digitales para la educación que tiene tres objetivos: permitir que cada 'líder' lleve innovación a sus escuelas; crear tecnología que 'empodere' a todos los educadores; equipar a cada estudiante asegurando la inclusión y la accesibilidad donde quieren que estén ${ }^{4}$. Workspace for Education tiene una edición gratuita y tres de pago, que se dividen en diversos espacios de actuación: Colaboración (Documentos, Presentaciones, Hojas de Cálculo, Drivercálculo, Drive, Formularios y Jamboard); Productividad (Google Classroom y Tareas); Comunicación (Gmail, Google Meet y Chat); y Organización (Keep y Calendar).

Google Classroom, el apartado específico de Workspace for Education, es una plataforma digital gratuita para docentes y estudiantes con la finalidad de desarrollar clases en modalidad no presencial que permite gestionar cursos, distribuir tareas, enviar comentarios dentro de un mismo espacio y entrelazar otras herramientas EdTech con Google. La "Application Programming Interface" (API) de Google Classroom, es el conjunto de procedimientos o lenguaje de programación que posibilita que otros softwares accedan a la plataforma digital. La API es como una "gramática estandarizada de funcionalidad" que facilita las "formas de intercambio e interoperabilidad" entre componentes programáticos y apps específicas y/o sistemas más grandes dentro de los que se encuentran (Snodgrass y Soon, 2019, p. 2). O, dicho de otro modo, la API es la "caja negra" de un lenguaje codificado que establece las "reglas de juego" para poder operar de modo interconectado en esta plataforma digital. Por haber pocas investigaciones al respecto, son necesarios, como sostienen Snodgrass y Winnie (2019), nuevos análisis que amplíen la descripción de las relaciones "entrecruzadas de las cuestiones sociales, técnicas y políticas de las API" (p. 3). Por ello es necesario seguir profundizando en los procesos de codificación y procesamientos algorítmicos de los datos de esta "lógica anticipatoria" en educación (Perrotta et al., 2021) que compone la gramática de la caja negra y las reglas de juego la innovación tecno-educativa de Google Classroom.

Junto a ello, dentro de Google for Education, se introducen también una serie de herramientas y software gratuitas para la educación incorporados en diversos servicios (Chromebooks, Google Cloud, Realidad Virtual y Aumentada). A lo cual ha añadido, a raíz del confinamiento por la pandemia global Covid-19, "Teach from Home", una iniciativa para la formación de las familias y un centro de "Capacitación y Asistencia” para la formación docente.

Capacitación y asistencia, la sección concreta de Google for Education, es un espacio de recursos educativos dividido en "centro de profesores", "desarrollo profesional docente" y "transformation center". En este espacio se condensan una serie de programas sistematizados de formación docente con unas certificaciones específicas, recursos formativos sobre las herramientas tecnológicas, finalidades de la formación docente para la innovación tecno-educativa y múltiples narrativas, vídeos e imágenes sobre la formación en redes de docentes en Google.

Por ser Google la corporación más relevante en la innovación educativa global y por la escasez de análisis en educación sobre la innovación educativa en las plataformas digitales es necesario preguntarse: ¿cómo está "innovando" Google en la práctica educativa y en la formación docente a través de sus plataformas digitales? El objetivo de la investigación que aquí presentamos es analizar el relato que sobre innovación educativa de las prácticas educativas y de la formación docente vende Google a través de sus plataformas digitales corporativas. Este objetivo está enmarcado dentro de una investigación más amplia que intenta comprender los cambios de la tecnología digital y de los actores políticos que están liderando la transformación de la gobernanza educativa global (Perrotta et al., 2021) .

Tras esta introducción, el artículo se divide en tres apartados. En el primer apartado se detalla y se sistematiza la metodología de la investigación, donde se hibridan dos enfoques metodológicos: la tecnografía (Bucher, 2018) y la etnografía digital (Pink et al., 2016). El segundo apartado presenta los resultados de la investigación. Por un lado, se presentan los resultados que están centrados en el análisis de la innovación tecno-educativa que ofrece la plataforma digital Google Classroom. Por otro lado, se presentan los

4 https://blog.google/outreach-initiatives/education/learning-with-google/

5 Redes políticas de gobernanza filantrópica: corporaciones, gobiernos y donantes ensamblando, movilizando y mutando las políticas en educación» (REPOGOFI) (Referencia: PPJIA2019-07) 
resultados que están centrados en el análisis de la formación docente para la innovación tecno-educativa de Google en educación. En el último apartado se presentan la discusión y las principales conclusiones de la investigación.

\section{Método}

En esta investigación se incorpora una nueva metodología de análisis al conectar dos enfoques metodológicos cualitativos hacia lo digital: la tecnografía (Bucher, 2018) y la etnografía digital (Pink et al. 2016). En el presente apartado se detalla y se sistematiza cómo hemos investigado "lo digital" como: método de análisis, objeto de análisis y campo de análisis. Para ello, se ha dividido este apartado metodológico en dos fases: La innovación tecno-educativa de Google Classroom como método, objeto y campo; y La formación tecno-educativa de Google for Education como método, objeto y campo. En ambas fases se expresan tanto el enfoque metodológico, las categorías de análisis, los instrumentos de información, el análisis de los datos, como la temporalización del proceso de indagación.

\subsection{Fase 1. La innovación tecno-educativa de Google Classroom como método, objeto y campo}

Lo digital como método. El método de análisis de esta fase está basado en la tecnografía. La tecnografía es un método de acercamiento analítico que se fundamenta en la hibridación de elementos de análisis etnográficos con elementos más propios del análisis tecnológico (Burch, 2018; Jansen y Vellema, 2011). Sirve porque analiza e interpreta lo tecnológico "para examinar la interacción entre un conjunto diverso de actores (tanto humanos como no humanos)" (Bucher, 2016, p. 86). Es, en sí, parte de la "ciencia social descriptiva de la tecnología que examina la interacción humano-máquina/herramienta" (Jansen y Vellema, 2011, p. 169). Es útil para examinar los mecanismos de poder y control de los códigos, de los algoritmos y de los datos. Es decir, es un acercamiento metodológico que sirve para adentrarse en la "caja negra" de las plataformas digitales para ver la capacidad de agencia de los softwares y como dice Bruno Latour (2005) "para hacerlos hablar, es decir, hacerlos ofrecer descripciones de sí mismos”, para comprender lo que "hacen hacer a otros, humanos y no humanos" (p. 79).

Lo digital como objeto de estudio. El objeto de investigación de la indagación tecnográfica es la plataforma digital de Google Classroom. Por un lado, se examina el funcionamiento tecnográfico de la API de Classroom mediante el análisis de los códigos, los algoritmos y los datos a través de la información datificada de Google. Lo que se hace en sí, mediante la tecnografía de Google Classroom, es adentrarse en el funcionamiento de los códigos y el procesamiento algorítmico de los datos para comprender las operaciones y los lenguajes tecnológicos de la innovación educativa de Google que cambian las prácticas tecno-educativas. Como en todo análisis tecnográfico, hay que comenzar "preguntando qué sugiere el software en sí" (la API de Classroom en nuestro caso) para posteriormente comprender las "normas y valores que se han delegado y materializado en la tecnología" (Bucher, 2016, p. 86). Por otro lado, se analizan aspectos de la economía política de lo digital a través de las dinámicas de extracción de los datos y las nuevas relaciones rentistas que genera Google Classroom con la industria EdTech. Las preguntas de investigación son las siguientes: ¿Cómo funcionan los aspectos tecnográficos (códigos, operaciones y lenguajes) de la API de Google Classroom? ¿Cómo se genera el control tecno-educativo a través de la innovación de Google Classroom? ¿Cómo se utiliza la información extraída de los datos y las políticas de privacidad de Google Classroom?

Lo digital como campo. La plataforma digital de Google Classroom es, en sí misma, el campo en el que recolectamos los datos. Específicamente recolectamos y analizamos la documentación digital de la API de Classroom, la documentación digital de las políticas de privacidad de los datos y la información expuesta en la web sobre las apps que se entrelazan con esta gramática como nuevos espacios de rentismo para la industria EdTech. Se ha sistematizado la información de cara a organizar los registros de los documentos de la API de Classroom, los links y los documentos de las políticas de privacidad de los datos en Google a través del software de análisis de datos cualitativos QSR NVivo12. Junto a ello, se ha utilizado la herramienta "archive.org" para descargar toda la información de las webs referida a los registros y hacer diversas entradas para registrar las modificaciones. La organización de la información registrada se ha hecho mediante el software NVivo12 dada las posibilidades que ofrece para codificar y posteriormente analizar 
la información. De este proceso han emergido tres categorías inductivas, cuyo desarrollo se presenta en el apartado "resultados": 1) control tecno-educativo de Google Classroom y 2) Privacidad de los datos y 3 ) Rentismo de los datos. Estas tres categorías hacen emerger la categoría principal: Innovación tecno-educativa de Google Classroom.

\subsection{Fase 2. La formación tecno-educativa de Google for Education como método, como objeto y como campo}

La digital como método. La etnografía digital se utiliza aquí como parte de un enfoque metodológico para indagar en la relación entre los elementos digitales, sensoriales, atmosféricos y materiales de nuestros entornos (Pink et al., 2016). No se trata simplemente de trasladar las estrategias de la tradición etnográfica, muy propia de los estudios antropológicos, a los elementos cotidianos con los que interactuamos y son partes de nuestra "sociedad digital". Lo que buscamos con el acercamiento de la etnografía digital es comprender los cambios sociales a través de los significados que los discursos de las plataformas digitales atribuyen al mundo. Es útil para comprender parte de los "imaginarios socio-técnicos", como esas "formas de vida social y orden social imaginadas colectivamente que se reflejan en el diseño y cumplimiento de proyectos científicos y/o tecnológicos específicos" (Jasanoff y Kim, 2009, p. 120). Es decir, en cada cambio digital siempre hay una técnica que opera de modo objetivo mediante unos mecanismos precisos que son de carácter analítico, pero simultáneamente hay una proyección de los cambios sociales o educativos, en este caso, que son desarrollados por actores que tienen unos intereses y visiones específicas sobre el tipo de sociedad que pretenden construir.

Lo digital como objeto. La plataforma digital de Google for Education es, en sí misma, el objeto de la indagación de esta etnografía digital. Por un lado, se analizan las narrativas digitales de la formación docente tecno-educativa de carácter innovador por parte de Google y la cultura digital que Google quiere proyectar respecto al profesionalismo docente. Por otro lado, se analizan los aspectos de marketing de innovación educativa que la corporación pretende proyectar a través de la cultura de formación docente que intentan expandir por todo el mundo. Para ello, buscamos responder a las siguientes preguntas: ¿cómo se presenta la formación docente innovadora a través de los espacios digitales de Google for Education?, ¿qué discursos proyecta Google respecto a los significados del profesionalismo docente y la formación tecno-educativa?, ¿mediante qué lógicas de mercado genera la formación docente Google?

Lo digital como campo. La plataforma digital de Google for Education es, a su vez, el campo de análisis. Mediante una observación no participante, examinamos y recolectamos los datos a través de la información extraída de los discursos, las imágenes y los vídeos que se exponen en los diferentes espacios digitales de Google for Education para comprender la construcción de significados sobre la innovación y el profesionalismo en la formación docente. Concretamente, extraemos los datos de los análisis del apartado "capacitación y asistencia", del manual de desarrollo profesional docente Google, de las webs de la comunidad educativa global Google y del espacio denominado "transformation center". Los registros de los documentos de Google, las webs, los links, las imágenes y los vídeos se sistematizan con NVivo12. Se utiliza "archive.org" como herramienta para descargar toda la información de las webs referida a los registros y hacer diversas entradas para examinar las modificaciones. El conjunto de webs analizadas son las que forman parte de la plataforma digital de la corporación que tienen relación con la formación docente y los datos (Google, 2021a; Google, 2021b; Google, 2021c; Google, 2021d; Google, 2021e; Google, 2021f; Google, 2021g; Google, 2021h). Se realiza la descarga de vídeos mediante el software "4K Video Downloader". Para registrar la descarga de imágenes completas de las webs a mayor velocidad y de modo completo se utiliza la herramienta "ImageCyborg". Las categorías de análisis son inductivas y emergen de los datos analizados mediante criterios interpretativos. Concretamente, generamos la codificación interpelando a dos categorías que se expresan en los resultados: 1) profesionalismo docente de Google y 2) Formación docente innovadora de la Comunidad Google. Estas dos categorías generan la categoría general: Innovación de la formación docente tecno-educativa de Google for Education.

\subsection{Temporalización}

Todos los procesos, registros y sistematización de los datos del análisis que se tienen en cuenta en las dos fases de investigación se recogen y analizan desde comienzos del mes de marzo de 2020 (momento en el que la Organización Mundial de la Salud declara la pandemia global Covid-19) hasta el 1 marzo de 2021. El 
punto de partida se sitúa en la declaración de la pandemia global dado que Google comienza a expandir sus actividades como medio para posibilitar la enseñanza no presencial. Si bien el campo de análisis ha sido constante, la interacción de los registros en archive.org se desarrollan cada tres meses: junio 2020, septiembre 2020, diciembre de 2020 y febrero de 2021, con el fin de detectar cambios significativos en las webs analizadas.

\section{Resultados}

En este apartado se presentan, primero, los resultados del análisis tecnográfico de la API de Google Classroom. Y segundo, los resultados de la etnografía digital de la formación docente en Google for Education.

\subsection{La innovación tecno-educativa en la "caja negra" de Google Classroom}

La API de Google Classroom se presentó en el prestigioso evento de la industria educativa tecnológica "International Society for Technology in Education" (ISTE) en Filadelfia en 2015. Zach Yeskel, el gerente que la presentó en ISTE como una herramienta que facilitaría la integración de la tecnología de Google para ayudar a la docencia ${ }^{6}$, llevaba en su camiseta el lema que quería transmitir "Less tech-ing, More teaching". Ya había mostrado este espíritu de innovación educativa el mismo Zach Yeskel en 2014 cuando presentó Classroom, en el blog de Google, pero con el lema invertido: "More teaching, Less tech-ing"'. El logotipo de la camiseta, que se ha convertido en un icono del emblema de Google en educación, "Menos tecnología/más enseñanza", está implícito también el discurso principal con el que se patrocina Google Classroom:

Obtenga más tiempo para enseñar e inspirar a los alumnos con Classroom. Una herramienta fácil y gratuita que ayuda a los educadores a administrar y evaluar el progreso de manera eficiente, al tiempo que mejora las conexiones con los alumnos de la escuela, el hogar o mientras viajan. (Google, 2021a)

\subsubsection{Control tecno-educativo de Google en la caja negra de Classroom}

El control educativo de Google con la API de Classroom comienza al establecer unos parámetros de acceso y códigos específicos que sirven como dirección de protocolos y marcos de actuación para la innovación tecno-educativa. Para que los desarrolladores de software y la comunidad educativa puedan acceder a los datos de Classroom, lo que hace Google es controlar el acceso a la API mediante un token (Google, 2021b). El token de Google Classroom es una firma cifrada o identificador del usuario para acceder y participar en la gramática preestablecida bajo la autorización de la corporación. Con ello, se permite a los administradores de software proporcionar cursos mediante una programación predeterminada, sincronizar los sistemas de información de los estudiantes con Classroom y obtener una visibilidad de las tareas de docentes y estudiantes que se realizan en su dominio. De ese modo, los administradores del sistema tienen un control del proceso educativo y de la información expuesta en la plataforma al regular la entrada a docentes, tutores y estudiantes, $\mathrm{y}$ así conocer todo lo que sucede en el proceso educativo preestablecido.

La gramática que proporciona la API de Google Classroom está conectada con el espacio de Workspace for Education a través del mismo lenguaje de codificación que controla y circunscribe la docencia a los dominios de Google. Es decir, aunque la API es una plataforma independiente que tiene su propio lenguaje, establece un marco de regulación y control respecto a la docencia, al hacer que el profesorado programe la enseñanza interconectado sus clases con otras herramientas de Workspace como "docs", "chromebooks", "Cloud" y otras apps que forman parte del domino de Google. Se genera así un control de las tareas docentes que lo "venden" como una herramienta que ayuda a los educadores para que puedan dedicar "más tiempo para enseñar e inspirar a los alumnos" (Google, 2021a).

El control educativo sobre el alumnado se realiza a través de un Sistema de Información de Estudiantes (SIS, por su traducción en inglés) donde se almacena toda la información sobre las tareas y el procesa-

6 https://www.youtube.com/watch?v=WgDpdezzchQ

7 https://cloud.googleblog.com/2014/08/more-teaching-less-tech-ing-google.html 
miento de los datos de cada estudiante. Es un almacenamiento de datos individuales donde se compilan los accesos, las asistencias, las tareas, las evaluaciones y todas las interacciones que un estudiante realiza en la plataforma digital. La API permite que puedan acceder administradores del sistema y tutores/familias de modo rápido a toda esa información. Permite también bloquear contenido e informar a docentes y familias sobre comportamientos sospechosos o información expuesta inadecuada que pueda contener aspectos sobre contenido engañoso, sexual, violento, de abuso infantil o de incitación al odio. Estos procesos de control se desarrollan a través de una jerarquía, ya que, si todos pueden controlar los datos de estudiantes, los desarrolladores de software y la corporación son los que pueden controlar los usos que hacen docentes y tutores de la plataforma (Google, 2021c).

\subsubsection{Modelo de negocio y dinámica extractiva del uso de los datos}

El control de los códigos y de los algoritmos de los datos procesados de la API de Google Classroom se rigen en su "Centro de privacidad y seguridad", donde manifiestan el alto "compromiso de crear productos que ayuden a proteger la privacidad tanto de alumnos como de profesores y que le brinden a tu

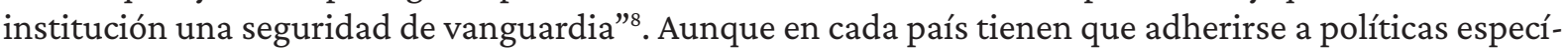
ficas, sostienen que el uso de los datos de la plataforma digital cumple rigurosos estándares, protocolos y políticas de protección de datos globales: Ley de Derechos Educativos y de Confidencialidad Familiar de 1974; Ley de Protección de la Privacidad Infantil en Internet de 1998; Promesa de Privacidad de los Alumnos presentada por el Foro sobre el Futuro de la Privacidad; Reglamento General de Protección de Datos 2016/679 de la Unión Europea; y el “Privacy Shield” entre Unión Europea y Estados Unidos. Pero más allá de los esfuerzos que la corporación realiza para proveer y proyectar seguridad, recientemente se concluyó la sentencia de un pago de 170 millones de dólares a la Comisión Federal de Comercio de Estados Unidos por infracciones a la Ley de Privacidad Infantil en Internet por el uso comercial de los datos'. La información para conseguir el consentimiento de las familias o tutores de Google Classroom ${ }^{10}$, manifiesta también que los datos recabados no serán utilizados con el fin de "orientar la publicidad" ni serán divulgadas con empresas, organizaciones o particulares sin consentimiento.

No obstante, sus políticas de privacidad y uso de los datos funcionan a través del modelo extractivo tradicional (Regan y Jesse, 2019), que comienza una vez que un usuario "sale" del aula para acceder a otras aplicaciones integradas de Google. Es decir, los anuncios llegan a los usuarios educativos cuando interactúan con "servicios adicionales" que operan con el lenguaje de la API de Classroom como YouTube, Google Maps y Blogger, entre otros ${ }^{11}$. Para Srnicek (2016) el uso de los datos de Google en educación se enmarca en una estrategia empresarial precisa de modelación de conducta de consumo. Esta práctica la consigue a través de la lealtad a la marca y la familiarización con la infraestructura existente. Por ello, si bien algunas de las características extractivas de Google son detectables en las especificaciones legales de sus políticas de privacidad, muchas otras están ocultas por la retórica de la documentación de Google orientada al cliente. Porque, "el objetivo retórico de los documentos de políticas orientados al cliente de Google es disfrazar el modelo de negocio y persuadir al lector para que entienda a Google como un servicio público gratuito, divorciado de los contextos y preocupaciones del mercado" (Lindh y Nolin, 2016, p. 650).

\subsubsection{Rentismo con la industria tecnológica de la innovación educativa}

Para proseguir en el análisis tecnográfico de la innovación educativa de la API de Google Classroom, en lo que sigue se señalan algunas de las nuevas "relaciones rentistas" (Birch, 2019; Komljenovic, 2021; Sadowski, 2019) que genera la plataforma con la industria EdTech. La plataforma de Classroom no funciona solamente con las diversas herramientas que ofrece Google, sino que está interconectada con cientos de apps que tienen que ser programadas con la gramática de Classroom (Google, 2021d). Estas apps que

\footnotetext{
8 https://edu.google.com/why-google/privacy-security/

9 https://www.ftc.gov/news-events/press-releases/2019/09/google-youtube-will-pay-record-170-million-allegedviolations

10 https://support.google.com/a/answer/7391849

11 https://support.google.com/ads/answer/2662856?co=GENIE.Platform\%3DAndroid\&hl=en
} 
funcionan con Classroom se activan con modelos de pago a través de diversas relaciones o suscripciones, tras la autorización que genera Google para que puedan interoperar con la plataforma.

La gramática de Classroom genera este tipo de relaciones rentistas ofreciendo y promocionando otras herramientas y apps que conecta la API con otros "EdTech Unicorns" a la vez que compiten directamente con otras corporaciones. Por ejemplo, Google establece una relación con Coursera (EdTech Unicorn valorada con 2.5 billones de dólares) que se encarga de la formación y certificación en Google Classroom. También con "Vidyartha", una app donde se incorporan todas las funcionalidades de Google Classroom con las visualizaciones en clase del gigante tecnológico indio BYJU'S (corporación valorada con 12 billones de dólares). Un ejemplo de competencia directa es el que realiza a Duolingo, otro "EdTech Unicorn" valorado en 2.4 billones de dólares, mediante el lanzamiento de "Rivet" ${ }^{2}$, una app para aprender inglés mediante la lectura en voz alta que utiliza diversos mecanismos de Inteligencia Artificial en educación. Estas operaciones que ofrece la API de Classroom con otras industrias tecnológicas son solo algunos ejemplos de las nuevas relaciones rentistas en educación de intermediarios y competencias que comienzan a aumentar en las agendas educativas globales (Komljenovic, 2021).

\subsection{Etnografía digital de la formación docente en la innovación tecno-educativa de Google for Education}

El espacio específico dedicado a la formación docente de Google for Education, denominado "Capacitación y Asistencia", pone de manifiesto la importancia que tiene la formación docente dentro de la estrategia de innovación tecno-educativa de Google. La multinacional tecnológica sostiene que Workspace for Education es, en sí, una plataforma que: "transforma la manera en que los educadores y los alumnos innovan, aprenden y trabajan” (Google, 2021e).

\subsubsection{Desarrollo profesional piramidal}

Google asegura que con su "Centro de profesores" puede convertir a las escuelas en "centros innovadores" a través de sus herramientas y las capacitaciones que imparte ya que "el aprendizaje innovador empieza con una sólida capacitación de los educadores”. La capacitación desarrollada por Google se organiza como un proceso de desarrollo profesional docente de superación de pruebas, donde el docente debe ir demostrando el dominio de las herramientas de Google, evidenciando su compromiso mediante certificaciones, y captando, a su vez, nuevos docentes para que sean formados en estas herramientas y expandan las narrativas y las herramientas de la corporación por todos los espacios educativos posibles.

La iniciación en este proceso de formación implica obtener la certificación como "Educador" Google. Para ello el docente se tiene que empoderar con capacitaciones online, a través de una formación continua en el manejo de la tecnología de Google que evidencien su capacidad para ser un docente innovador. Cada docente tiene que validar su "competencia" en el uso de los productos de Google, pagando por realizar un examen que da una certificación válida para tres años. Luego hay que volver a pagar para revalidar la certificación. La ascensión en este camino de 'sacrificio' de la innovación comienza "logrando" las certificaciones de educador de nivel 1 y 2 , y posteriormente pudiendo convertirse en "capacitador certificado" de Google, donde se puede pasar posteriormente a "dirigir y capacitar a otros educadores con tu experiencia en las herramientas de Google" (Google, 2021e).

Para esa tercera fase de expansión (capacitador certificado) los nuevos docentes Google tienen que utilizar su "experiencia" y "liderazgo" con el fin de convencer a otros docentes para que entren en la "Comunidad Google" y transformen sus aulas también con las herramientas de la corporación. Impulsan que se "destaquen" como líderes y amplíen "el impacto sobre otros educadores y sus alumnos". Bajo esta lógica, la corporación pretende ir cooptando al profesorado "líder" y "reclutando" alumnado también "líder" que "amen la tecnología" y se conviertan en motivadores y coach de este cambio, "dándoles la oportunidad de brillar y desarrollar habilidades de liderazgo mientras ayudan a su comunidad escolar". Proponen incluso, en esta lógica del "Transformation Center", que se les "ofrezca incentivos a los estudiantes por su participación”.

12 https://rivet-better-reading-practice.uptodown.com/android 
Todo ello está enmarcado en un proceso de inmersión ideológica progresiva, que identifica parte de la construcción de significados que Google ofrece a la formación: "Graba un video como capacitador (...) En el primer minuto, sorpréndenos explicando por qué quieres ser Capacitador, qué te hace singular y por qué tienes el estilo de Google". Y para ser "capacitador" certificado se tiene que buscar clientes y expandir el negocio, dando sesiones una vez al mes, que deben ser comprobadas, además de examinarse periódicamente sobre la última versión instalada del producto.

La ascensión final en la carrera de desarrollo profesional docente de Google es alcanzar certificación de "Innovador Certificado". Para ello hay que lanzar "un proyecto único que te apasione en el que uses tecnología para resolver desafíos en la educación". Una vez superada esta prueba pueden unirse a la "comunidad de innovadores" y recibir "asesoramiento personalizado" por parte del "Transformation Center" de Google (Google, 2021f).

Todo este proceso ha generado, a su vez, otra red rentista de empresas y negocios de la industria EdTech educativa privada dedicada a la formación docente para Google. Son relaciones que se generan con lo que han denominado "Google for Education Parnerts" (Google, 2021g) cuya finalidad pretende ser construir un "proyecto pedagógico que triunfe de la mano de las nuevas tecnologías educativas". Incluso Google ofrece a cada docente una 'capacitación' "con un experto capacitador individual" o "un socio de desarrollo profesional certificado de tu zona para obtener capacitación y asistencia en persona o virtual".

Las cuatro fases de ascensión en el profesionalismo docente de Google (Educador 1, Educador 2, Capacitador e Innovador), como se puede ver en la Figura 1, son certificaciones que garantizan para Google que quien las obtiene es un innovador educativo que asume y expande la construcción de significados de la formación docente de la corporación.

Figura 1

Certificaciones Google

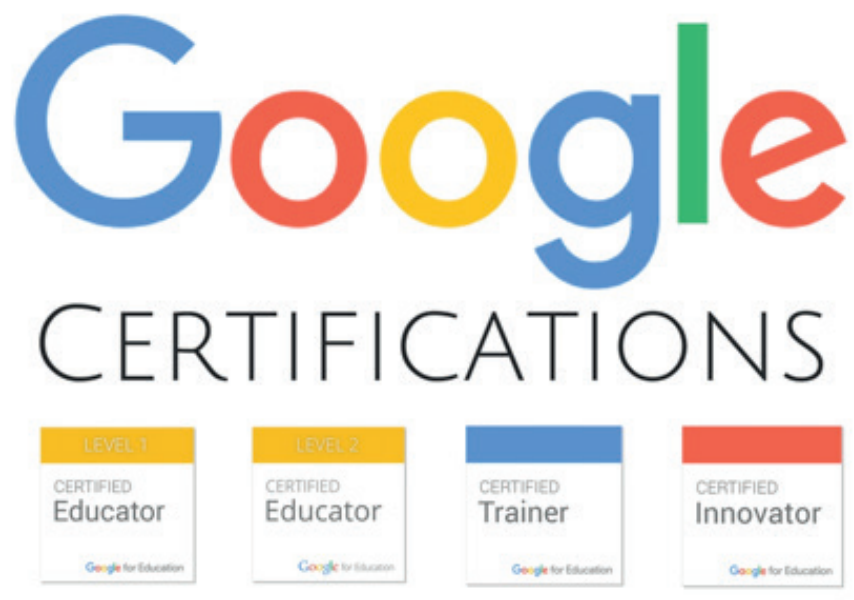

Nota. Recuperado de Google (2021f).

Todo ese esquema de ascensión en la formación docente certificada, denominado desarrollo profesional Google, opera con un mecanismo similar a los esquemas de mercado multinivel o piramidal, donde se recluta, educa y motiva a los nuevos consumidores (Groß y Vriens, 2019). En el esquema piramidal de la formación de Google, los participantes certificados tienen que recomendar y captar a más docentes con el objetivo de que los nuevos participantes produzcan beneficios a los participantes originales. Al entender y asumir la construcción de significados sobre la formación docente en este desarrollo profesional, la corporación puede, a su vez, extender su lógica de mercado a través de la innovación tecno-educativa. Este esquema de superación de pruebas en la formación y desarrollo del docente no solo reemplaza la labor de formación permanente de las administraciones educativas públicas. También supone una actualización del discurso neoliberal del "optimismo cruel" (Moore y Clarke, 2016) para responsabilizar al docente de su proceso de desarrollo profesional, mediante la exigencia de acumular 'éxitos' constantes, dinámica propia de la desprofesionalización docente. 


\subsubsection{Comunidad educativa Google}

La formación docente para la innovación tecno-educativa de Google no tiene solo una vertiente individual. Mediante un sistema de creación de redes, que se extienden constantemente y convierte a los participantes en patrocinadores que defienden los productos de la corporación, la formación innovadora de Google se teje también a través de la "Comunidad Google".

Esta Comunidad se expande mediante redes de 'docentes innovadores' denominadas Grupo de Educadores Google (GEG) ${ }^{13}$. La finalidad de los GEG es "compartir, colaborar y respaldarse mutuamente", conectando a "educadores apasionados para discutir los beneficios de la tecnología en escuelas, aulas y comunidades de todo el mundo" y "un foro para que los educadores colaboren, compartan y se apoyen entre sí" (Google, 2021h). Cada grupo tiene como organizador a un voluntario local, que es el "Líder del GEG”. La Comunidad Google está conformada por más de cuatrocientos de GEG en países de todos los continentes (Figura 2).

Figura 2

Google Educator Groups

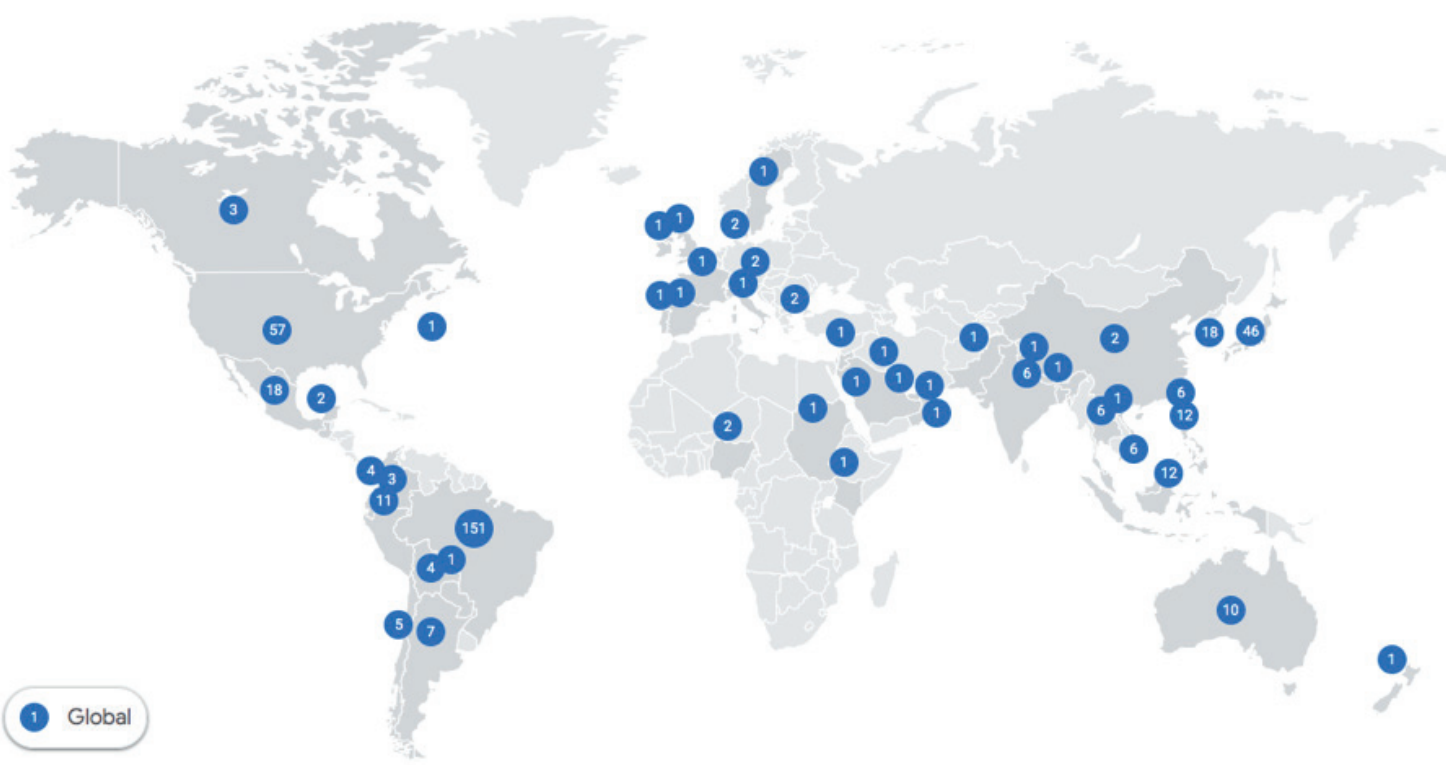

Nota. Recuperado de Google (Google, 2021h)

Las GEG tienen sus "Centros de Profesores”, sus "Cursos de capacitación”, sus "guías de productos", sus "Certificaciones" y sus "Programas para Capacitadores Certificados" y para "Innovadores Certificados", donde se "apoya a los mejores educadores que están entusiasmados por crecer profesionalmente, abogan por la tecnología e innovan para mejorar las aulas, las escuelas y las comunidades locales" (Google, 2021f). Estas redes auto-organizan eventos y jornadas para motivar a seguir implicándose de forma activa en este proceso de formación tecno-educativo bajo el lema: "Interactúa, inspírate y aprende de una comunidad de educadores apasionados".

Cada GEG tiene su propia guía y código de conductas que refleja las directrices aprobadas por la comunidad globa $1^{14}$. En los análisis de las guías se ve la uniformidad de estas directrices: desde cómo hacer un presupuesto, a buscar socios, patrocinadores y alianzas instituciones para apoyar el evento, o elegir las decoraciones, los preparativos, alquilar materiales y contratar al personal de apoyo. En los eventos el profesorado de cada red tiene que exponer testimonios, experiencias, casos de éxito y debate sobre cómo expandirse a través del estilo Google. En ellos se presentan vídeos promocionales hechos por "profesores

13 https://edu.google.com/latest-news/communities/

14 Ver, por ejemplo, diferentes GEG: https://www.globalgeg.org/globals-beginner-guide;https://sites.google.com/ view/geguk 
Google” con "sonrisas Google”, “camisetas Google”, "lenguaje Google” y toda una serie de productos de marketing: "talleres de compromiso profesional" e "insignias" o badges (como los boys scouts) que se "coleccionan".

Cada participación en los eventos de auto-formación que desarrollan las GEG tiene que dejar evidencias a través de vídeos y testimonios de experiencia que son validadas por el responsable de cada taller del evento. Incluso, la formación online prosigue tras ver los vídeos de los talleres mediante tareas que exigen testimonios fotográficos de las mismas, responder preguntas o rellenar formularios para conseguir el diploma de participación y lograr así las insignias Google.

En todos los discursos analizados, se entremezcla constantemente las narrativas tecno-educativas con una terminología propia del marketing y un lenguaje que quiere establecer un "estilo Google". En el último evento que desarrolla la comunidad educativa global de Google antes del 1 de marzo de 2021 -que se lleva a cabo el 28 de febrero por el GEG de España- se puede observar esa interacción discursiva entre las narrativas tecno-educativas con las de marketing: "Una vez vistos los vídeos, consigue la insignia especial Google Innovators (Compromiso profesional) a través del siguiente formulario, pégala en tu pasaporte y atento/a a los sorteos que realizaremos en la clausura del GEGAndalucía On Tour!”15

\section{Discusión y conclusiones}

La innovación en la práctica educativa y en la formación docente que lidera Google a través de sus plataformas digitales se vende bajo un relato de innovación tecno-educativa. Como parte de las discusiones de investigaciones previas sobre los cambios sociales de la plataformización de la sociedad (Van Dijck et al., 2019) y del capitalismo de plataformas (Srnicek, 2016), que dominan las grandes corporaciones tecnológicas, Google está liderando este relato de la innovación educativa global a través de sus plataformas digitales. Como se ha demostrado previamente, la plataformización de la educación que desarrolla Google genera nuevas infraestructuras, avances socio-económicos y políticos que proyectan nuevos imaginarios socio-técnicos a través de sus plataformas digitales (Decuypere et al., 2021; Perrotta et al., 2021). Tal y como sucede en otros esferas socio-económicas (Birch et al., 2020), las plataformas digitales de Google en educación se sostienen como nuevos espacios de innovación que están generando relaciones rentistas de los datos anidando otras apps a sus términos de uso, produciendo un aumento de la gobernanza en el sector educativo y operando bajo nuevas tensiones entre la competencia y el monopolio en los mercados de educación digital (Komljenovic, 2021). Al igual que funcionan los discursos del solucionismo tecnológico que elaboran las corporaciones tecnológicas (Morozov, 2015), Google opera en la formación docente bajo nuevas narrativas que interrelacionan la innovación docente con el solucionismo de la tecnología que ofrecen en sus plataformas digitales.

En esta investigación, los resultados analíticos del funcionamiento tecnográfico de la API de la plataforma digital de Google Classroom manifiestan el afán de la innovación tecno-educativa de la corporación tecnológica. Todos los accesos y los procesos educativos que se desarrollan en Classroom son informaciones muy valiosas que son datificadas y procesadas bajo el dominio de la corporación tecnológica. Classroom opera reconfigurando nuevos mecanismos de control en educación a través de códigos, datos y algoritmos que generan unas prácticas educativas programadas y predeterminadas. Al crear la API mediante accesos, autorizaciones y restricciones, lo que hacen los desarrolladores de software de Google es codificar y programar los procesos de enseñanza-aprendizaje para anticiparse al proceso educativo. Este nuevo lenguaje predefinido y anticipado en la "caja negra" de Google Classroom genera procesos de poder y control en la innovación tecno-educativa que no existían antes del comienzo del uso de las plataformas digitales en educación. Las políticas de privacidad y el uso de los datos de usuarios en Google Classroom operan mediante un modelo extractivo cuando la plataforma se interrelaciona con otras herramientas y softwares de la industria EdTech.

Aquí se demuestra que las relaciones rentistas se generan mediante una red de apps y herramientas, vinculadas a la gramática de Classroom, que imponen a los sistemas educativos un modelo, presenta-

15 https://sites.google.com/gedu.es/gegspain-andalucia/encuentros/geg-andalucia-on-tour-2021/d\%C3\%ADa-deandaluc\%C3\%ADa-28-de-febrero 
do como innovador. Estos procesos rentistas de la innovación tecno-educativa de Google se enmarcan bajo procesos de producción y consumo marcadamente conductistas, cuyo valor no se cifra para estas compañías en la innovación educativa sino en las nuevas oportunidades que ofrecen para capturar valor añadido a través de la extracción masiva de datos. El rentismo que genera Google está promoviendo nuevas relaciones de dependencia con una industria educativa global que está transformando las agendas educativas globales.

El análisis del espacio digital "Capacitación y Asistencia", de Google for Education, demuestra el afán tecno-educativo que tiene Google respecto a la formación docente. La corporación tecnológica sistematiza en su plataforma digital discursos, programas de formación y certificaciones docentes para construir nuevos significados de la innovación docente en la formación de profesorado. Google está proyectando relatos solucionistas que aseguran que la formación docente y el desarrollo profesional tienen que desarrollarse mediante la innovación que proporciona en sus plataformas. Los significados que elabora Google sobre la formación docente son parte de relatos solucionistas que argumentan la necesidad de transformar la educación a través de la tecnología digital como medios para erradicar las problemáticas educativas y modernizar los sistemas educativos. Como se ha demostrado, la corporación establece un avance en los nuevos imaginarios socio-técnicos, al proyectar formación docente con su tecnología a la vez que se genera las narrativas de cómo imagina al profesorado innovador.

La formación docente tecno-educativa de la corporación generada a través de la sistematización de programas de capacitación y "certificaciones Google” tienen el propósito de generar nuevos discursos sobre el profesionalismo docente. Lo que hace Google es construir un desarrollo profesional piramidal a través de superaciones de pruebas de evaluación, certificados que verifican la superación de la formación y compromisos para extender los propósitos de la corporación tecnológica entre más docentes y más espacios educativos. Estas estrategias son propias de la desprofesionalización docente.

Todo ello va aunado con la expansión de nuevos negocios educativos que se encargan de las certificaciones y de extender proyectos pedagógicos para generar escuelas innovadoras. Además, la formación docente tecno-educativa se desarrolla a través de redes de docentes de grupos de educadores Google por la mayoría de países que forman la "Comunidad Google". La comunidad de educadores expande las lógicas solucionistas de la corporación auto-organizando eventos y auto-formándose a través de sus centros de profesores y programas de capacitación que son certificados. Es probable que la comunidad de educadores siga expandiéndose, por lo que será necesario seguir analizando estos avances de la formación tecno-educativa de Google.

Como los resultados son parte de una investigación más amplia en curso, en el presente artículo hay dos limitaciones principales que, a su vez, representan futuras líneas de investigación. Por un lado, se puede observar que hay una escasez de resultados referidos a las relaciones rentistas y a los efectos de las políticas de privacidad de los datos que procesa la plataforma digital de Google para la innovación tecno-educativa. Por otro lado, la agenda de investigación requiere de entrevistas semi-estructuradas para interpretar cómo el profesorado en diferentes países está valorando la formación docente para la innovación tecno-educativa de Google. En este sentido, pretendemos en investigaciones posteriores avanzar más allá del análisis de las plataformas digitales e indagar y analizar también las miradas y puntos de vista de los involucrados en la formación docente y los resultados del proceso formativo en quienes están participando en el mismo.

\section{Referencias}

Birch, K. (2019). Technoscience rent: Toward a theory of tentiership for technoscientific capitalism. Science, Technology, \& Human Values, 45(1), 3-33. https://doi.org/10.1177/0162243919829567

Birch, K., Chiappetta, M. y Artyushina, A. (2020). The problem of innovation in technoscientific capitalism: Data rentiership and the policy implications of turning personal digital data into a private asset. Policy Studies, 41(5), 468-487. https://doi.org/10.1080/01442872.2020.1748264

Bucher T. (2016). Neither black nor box: Ways of knowing algorithms. En S. Kubitschko y A. Kaun (Eds.), Innovative methods in media and communication research (pp. 81-89). Palgrave Macmillan.

https://doi.org/10.1007/978-3-319-40700-5_5

Bucher, T. (2018). If...then: Algorithmic power and politics. Oxford University Press. 
Couldry, N. y Mejias, U. A. (2019). Data colonialism: Rethinking big data's relation to the contemporary subject. Television \& New Media, 20(4), 336-349. https://doi.org/10.1177/1527476418796632

Decuypere, M., Grimaldi, E. y Landri, P. (2021). Introduction: Critical studies of digital education platforms. Critical Studies in Education, 62(1), 1-16. https://doi.org/10.1080/17508487.2020.1866050

Google. (2021a). Google classroom. https://edu.google.com/intl/en-eu/products/classroom/

Google. (2021b). API-classroom-authorizing requests. https://developers.google.com/classroom/guides/auth

Google. (2021c). API-classroom-manage. https://developers.google.com/classroom/guides/manage-guardians

Google. (2021d). Apps that work\#withClassroom. https://edu.google.com/intl/en-eu/products/classroom/apps/

Google. (2021e). Google for education. https://edu.google.com

Google. (2021f). Teacher center. https://edu.google.com/intl/en-eu/teacher-center/?modal_active=none

Google. (2021g). Google for education-partnes. https://edu.google.com/partners/

Google. (2021h). Google for education-communities. https://edu.google.com/latest-news/communities/

Groß, C. y Vriens, D. (2019). The role of the distributor network in the persistence of legal and ethical problems of multi-level marketing companies. Journal of Business Ethics, 156(2), 333-355.

https://doi.org/10.1007/s10551-017-3556-9_

HolonIQ. (2021). Global EdTech unicorns. https://www.holoniq.com/edtech-unicorns/

Ideland, M. (2021). Google and the end of the teacher? How a figuration of the teacher is produced through an ed-tech discourse. Learning, Media and Technology, 46(1), 33-46. https://doi.org/10.1080/17439884.2020.1809452

Jansen, K. y Vellema, S. (2011). What is technography? Wageningen Journal of Life Sciences, 57(3-4), 169-177. https://doi.org/10.1016/j.njas.2010.11.003

Jasanoff, S. y Kim, S.-H. (2009). Containing the atom: Sociotechnical imaginaries and nuclear power in the United States and South Korea. Minerva, 47(2), 119-146. https://doi.org/10.1007/s11024-009-9124-4

Komljenovic, J. (2021). The rise of education rentiers: Digital platforms, digital data and rents. Learning, Media and Technology. https://doi.org/10.1080/17439884.2021.1891422

Latour, B. (2005). Reassembling the social: An introduction to actor-network-theory. Oxford University Press.

Lindh, M. y Nolin, J. (2016). Information we collect: Surveillance and privacy in the implementation of google apps for education. European Educational Research Journal, 15(6), 644-663. https://doi.org/10.1177/1474904116654917

Means, A. J. (2018). Platform learning and on-demand labor: Sociotechnical projections on the future of education and work. Learning, Media and Technology, 43(3), 326-338. https://doi.org/10.1080/17439884.2018.1504792

Moore, A. y Clarke, M. (2016). Cruel optimism: Teacher attachment to professionalism in an era of performativity.Journal of Education Policy, 31(5), 666-677. https://doi.org/10.1080/02680939.2016.1160293

Morozov, E. (2015). To save everything, click here: The folly of technological solutionism. PublicAffairs.

Perrotta, C., Gulson, K. N., Williamson, B. y Witzenberger, K. (2021). Automation, APIs and the distributed labour of platform pedagogies in Google Classroom. Critical Studies in Education, 62(1), 97-113.

https://doi.org/10.1080/17508487.2020.1855597

Pink, S., Horst, H., Postill, J., Hjorth, L., Lewis, T. y Tacchi, J. (2016). Digital ethnography: Principles and practice. Sage.

Regan, P. M. y Jesse, J. (2018). Ethical challenges of edtech, big data and personalized learning: Twenty-first century student sorting and tracking. Ethics and Information Technology, 21(3), 167-179.

https://doi.org/10.1007/s10676-018-9492-2

Sadowski, J. (2019). When data is capital: Datafication, accumulation, and extraction. Big Data \& Society, 6(1), 20-54. https://doi.org/10.1177/2053951718820549 
Snodgrass, E. y Soon, W. (2019). API practices and paradigms: Exploring the protocological parameters of APIs as key facilitators of sociotechnical forms of exchange. First Monday, 24(2), 1-20.

https://doi.org/10.5210/fm.v24i2.9553

Srnicek, N. (2016). Platform capitalism. Cambridge Polity Press.

Tooley, J., Rudolph, J., Melnik, S. y Tan, S. (2020). Private schools for the poor as a disruptive educational innovation. An interview with Professor James Tooley.Journal of Applied Learning \& Teaching, 3(2), 136-149. https://doi.org/10.37074/jalt.2020.3.2.22

Van Dijck, J., Poell, T. y De Waal, M. (2018). The platform society: Public values in a connective world. Oxford University Press.

Williamson, B. y Hogan, A. (2020). Commercialisation and privatisation in/of education in the context of Covid-19. Education International press.

Zuboff, S. (2019). The age of surveillance capitalism: The fight for a human future at the new frontier of power. PublicAffairs.

\section{Breve CV de los autores}

\section{Geo Saura}

Profesor Ayudante Doctor en la Universidad de Granada. Fue Investigador Postdoctoral en la Universitat Autònoma de Barcelona (2015-2018) e Investigador Predoctoral (2012-2015) entre Universidad de Granada y University of Roehampton desarrollando su PhD internacional sobre políticas y neoliberalismo que recibió el Premio Extraordinario a la mejor Tesis Doctoral en Ciencias Sociales. Su investigación está centrada en la política educativa y en la neoliberalización de la educación. Está investigando en varios proyectos investigación competitivos y es Investigador Principal del Proyecto "Redes políticas de gobernanza filantrópica: corporaciones, gobiernos y donantes ensamblando, movilizando y mutando las políticas en educación” (REPOGOFI). Email: geosaura@ugr.es

ORCID ID: https://orcid.org/0000-0002-2365-9532

\section{Enrique Javier Díez Gutiérrez}

Doctor en Ciencias de la Educación. Licenciado en Filosofía. Diplomado en Trabajo Social y Educación Social. Ha trabajado como educador social, maestro de primaria, profesor de secundaria, orientador en institutos y responsable de atención a la diversidad en la administración educativa. Es Profesor de la Facultad de Educación de la Universidad de León. Especialista en organización educativa, desarrolla su labor docente e investigadora en el campo de la educación intercultural, el género y la política educativa. Entre sus publicaciones se encuentran: Educación crítica e inclusiva para una sociedad poscapitalista (Octaedro, 2021), Educación para el bien común (Octaedro, 2020) y La educación en venta (Octaedro, 2020) con Juan Ramón Rodríguez. La asignatura pendiente (Plaza y Valdés, 2020), La revuelta educativa neocon (Trea, 2019). Email: ejdieg@unileon.es

ORCID ID: http://orcid.org/0000-0003-3399-5318

\section{Pablo Rivera Vargas}

Profesor Lector del Departamento de Didáctica y Organización Educativa de la Universidad de Barcelona (España). Es miembro del grupo de investigación ESBRINA (2017SGR 1248) y del Instituto de Investigación en Educación de la Universidad de Barcelona. Doctor en Educación y Sociedad (Universidad de Barcelona) y en Sociología (Universidad de Zaragoza). Sociólogo y Máster en Ciencias Sociales (Universidad de Chile). Ha sido académico visitante en la Universidad de Wisconsin Madison (EE. UU.), en la Universitat Oberta de Catalunya (España) y en la Fundación Ceibal (Uruguay). Profesor Visitante en la Facultad de Educación y Ciencias Sociales de la Universidad de Andrés Bello (Chile). Su actividad investigadora se ha relacionado con políticas públicas de inclusión digital en contextos de aprendizaje formal y no formal. Email: pablorivera@ub.edu

ORCID ID: https://orcid.org/0000-0002-9564-2596 